\title{
Uso racional de antimicrobianos en el departamento de medicina interna de un hospital universitario: resultados de una experiencia piloto
}

\author{
Susana Cabrera A., Leonardo Sosa, Zaida Arteta, Verónica Seija, Soledad Mateos, \\ Abayubá Perna, Mariana Firpo, Gabriela Pereira, Gimena Lombardo, Ximena Añón, \\ Mariela Teliz, Natalia Danese, Maynés López, Jimena Bueno, Cecilia Romero, \\ Raúl Rozada, Virginia Antelo, Ana Laura Quijano y Djalma Oper
}

\section{Rational use of antibiotics in the department of internal medicine from a university hospital: results of a pilot experience}

Introduction: The programs of rational use of antibiotics are designed to optimize antimicrobial therapy and minimize the emergence of bacterial resistance. In order to optimize the use of antibiotics we implemented an educational program based on the application of a checklist criteria for the rational use of these drugs. Method: We performed a cohort study unpaired in the Department of Internal Medicine, during three months. We compared a prospective cohort (A) which used a checklist, with a retrospective cohort (B) in wich prescription was based on usual clinical practice. Results: We included 227 prescriptions of antibiotics. In cohort A compared to B, there was a higher proportion of switch to oral antibiotics agents and adjustment of the antimicrobial therapy to the susceptibility in the antibiogram and reduced use of associated antibiotics. Total antibiotic consumption was 117.7 DDD/100 bed-days (Defined Daily Doses). Consumption in cohorts A and B was 46.1 DDD/100 bed-days and 71.6 DDD/100 bed-days (reduction, 35.6\%). There was also a reduction in consumption of ceftriaxone, ceftazidime, quinolones, vancomycin and carbapenems. Costs were reduced by 55\%. There was no difference in the average hospital stay. Conclusions: The implementation of an educational strategy based on a checklist allowed the optimum use of antibiotics.

Key words: Antimicrobial agents, rational use of antibiotic.

Palabras clave: Antimicrobianos, uso racional de antimicrobianos.

\section{Introducción}

$\mathrm{E}$ l consumo de antimicrobianos corresponde aproximadamente al 30\% del consumo de fármacos en el hospital y se ha estimado que entre 20 y $50 \%$ de estas prescripciones son inadecuadas ${ }^{1,2}$. El uso excesivo o inapropiado de estos agentes es uno de los factores que contribuye al desarrollo y selección de cepas bacterianas resistentes $^{3-5}$. Se ha demostrado que estas cepas son responsables de mayor morbilidad, mortalidad, estadía hospitalaria y costos asociados ${ }^{6-10}$. Un trabajo de investigación llevado adelante en nuestro hospital demostró costos significativamente mayores para las infecciones causadas por Staphylococcus aureus resistente a meticilina (SARM) en comparación con Staphylococcus aureus sensible a meticilina ${ }^{11}$.

No todos los médicos consideran en toda su complejidad el concepto de "uso apropiado de antimicrobianos". Este concepto no sólo comprende la actividad que el antimicrobiano pueda tener sobre el agente causal sino que también debe incluir la dosis, la vía y el tiempo de administración.

El desarrollo e implementación de programas para el uso apropiado de antimicrobianos son prioritarios para optimizar la relación costo-eficacia y costo-eficiencia y contribuir a la contención de la resistencia bacteriana y disminuir $\operatorname{costos}^{12-14}$. Numerosos estudios han evaluado las estrategias propuestas en estos programas para uso apropiado de antimicrobianos tanto en el hospital como en la comunidad ${ }^{15-22}$.

Existen escasos estudios sobre consumo de antimicrobianos en Uruguay ${ }^{23,24}$. No hay hasta el momento, estudios publicados que evalúen estrategias para un uso adecuado de los mismos, si bien varias instituciones aplican medidas restrictivas.

El objetivo del presente trabajo fue implementar una estrategia para mejorar el uso de antimicrobianos en un área de los Servicios de Medicina Interna del Hospital

\author{
Universidad de la República. \\ Montevideo, Uruguay. \\ Facultad de Medicina \\ Hospital de Clínicas \\ Clínica Médica A (SM, XA, ML) \\ Clínica Médica B (SCA, LS, GP, GL, \\ $M T, N D, R R, A L Q, D O)$. \\ Clínica Médica C (MF, JB, CR). \\ Departamento de Laboratorio \\ Clínico, Sección Microbiología (VS). \\ Cátedra de Enfermedades \\ Infecciosas (ZA, VA). \\ Instituto de Neurología. Sección \\ Neuroepidemiología (AP)
}

Los autores declaran no tener conflictos de interés.

Recibido: 17 de noviembre de 2010 Aceptado: 27 de septiembre de 2011

Correspondencia a:

Susana Cabrera Alonso susanacabrera1@gmail.com 
de Clínicas y evaluar su impacto comparándola con otra área del mismo servicio hospitalario donde no se realizó intervención alguna.

\section{Material y Método}

El Hospital de Clínicas (HC) es un hospital universitario de alta complejidad. En el año 2008 hubo un total de 8.993 egresos hospitalarios, con una estadía media de 14 días.

Población: Estuvo constituida por todos los pacientes internados en el Departamento de Medicina Interna (DMI) del HC entre el 1 de julio de 2008 y el 30 de septiembre de 2008 y que recibieron antimicrobianos. El DMI comprende tres servicios universitarios con un total de 131 camas disponibles. Se excluyeron aquellos pacientes que recibieron tratamientos antituberculosos, antifúngicos, antivirales, antiparasitarios o que procedían de otro servicio con una internación mayor a 7 días donde hubieran iniciado o recibido antimicrobianos.

Diseño: Se realizó un estudio de cohortes no pareadas. Una cohorte prospectiva (desde ahora cohorte A), integrada por un grupo de pacientes elegidos al azar, en los que la prescripción de antimicrobianos se realizó en el marco de un programa educativo de uso racional de antimicrobianos (64 camas). Esta cohorte se comparó con otra (cohorte B), integrada por pacientes que recibieron antimicrobianos de acuerdo a la práctica clínica habitual (67 camas) en el mismo período de tiempo. La recolección de la información se realizó en forma prospectiva para la cohorte A y en forma retrospectiva para la cohorte B. Para integrar la cohorte B se identificaron las historias clínicas de pacientes que recibieron antimicrobianos a través del registro de altas.

El programa de uso racional de antimicrobianos consistió en la visita diaria por parte de un médico responsable que revisaba, junto a los médicos tratantes, las indicaciones de antimicrobianos. Se utilizó una lista de verificación (check list) aplicada a las prescripciones de antimicrobianos, la cual contenía una lista de criterios, previamente definidos por infectólogo y microbiólogo, para el uso de antimicrobianos:

- favorecer el empleo de terapia secuencial cuando estaba indicado;

- adecuar el tratamiento antimicrobiano a los resultados de los cultivos bacteriológicos, de-escalando siempre que fuera posible;

- promover la monoterapia en lugar de la asociación de antimicrobianos;

- cambio a un antimicrobiano alternativo cuando se prescribían cefalosporinas de tercera generación, ciprofloxacina, vancomicina o carbapenem;

- uso de aminoglucósidos en monodosis.
Se intercambiaron opiniones con los médicos tratantes considerando la aplicabilidad de las medidas propuestas.

Además se registraron en un formulario: fecha de ingreso y egreso, lugar de internación, edad, sexo, antecedentes personales y co-morbilidades, lugar de adquisición de la infección, tipo de infección, antimicrobianos utilizados (nombre, dosis, intervalos, días); resultados microbiológicos y antibiograma, condiciones del paciente al alta. Cuando el paciente recibió antimicrobianos debido a más de una infección se registró en formularios separados.

Se midió el consumo de antimicrobianos expresado en dosis diaria definida (DDD) cada 100 días-paciente, tipo de antimicrobianos utilizados, tiempo de uso total en días, tiempo de uso por vía intravenosa u oral, número de antimicrobianos usados por infección, uso de asociaciones, tiempo de uso de antimicrobianos asociados, paso a vía oral, adecuación al espectro antimicrobiano y perfil de susceptibilidad del microorganismo, uso de aminoglucósidos en monodosis, estadía media hospitalaria y costos.

Se utilizaron los valores de DDD del listado publicado y actualizado por el WHO Collaborating Centre for Drug Statistic Methodology ${ }^{25}$. La DDD se basa en el promedio de la dosis de mantenimiento diaria del fármaco utilizado en su principal indicación y es asignada por el Anatomical Therapeutic Chemical (ATC). El cálculo de dosis diaria definida (DDD)/100 días-cama se realizó con la siguiente fórmula: $\mathrm{DDD} / 100$ días-cama = consumo en mg durante el período/DDD en $\mathrm{mg} \times \mathrm{n}^{\circ}$ de días incluidos en el período $\mathrm{x} \mathrm{n}^{\circ}$ de camas $\mathrm{x} \%$ de ocupación.

Análisis estadístico. Se utilizó el paquete estadístico G-STAT. Para el análisis descriptivo se utilizaron como medidas de resumen: para las variables contínuas, media y desvío estándar; para las variables categóricas, proporciones. Para los objetivos analíticos se utilizaron según las condiciones de aplicación, el test de chi cuadrado, el test de T, el test de Mann-Whitney. Se utilizó un nivel de significación de a $=5 \%$.

Consideraciones éticas. El presente trabajo fue aprobado por el Comité de Ética del Hospital de Clínicas.

\section{Resultados}

Durante el período de estudio se incluyeron 227 prescripciones de antimicrobianos en 219 pacientes, 121 (55,2\%) hombres y 98 mujeres (44,8\%). El período de estudio fue de 92 días, el número de camas incluidas fue 64 en la cohorte A y 67 en la cohorte B. El porcentaje de ocupación durante el período estudiado fue $96 \%$.

La cohorte A (en que se aplicó lista de verificación) estuvo integrado por 107 pacientes y la cohorte B por 112 pacientes. La edad media y su desviación estándar 
en las cohortes A y B fue $59 \pm 20$ años y $60 \pm 20$ años, respectivamente (diferencia no significativa, $\mathrm{T}$ de student).

En la Tabla 1 se muestran y comparan las características de las dos cohortes.

En cuanto a la procedencia de los pacientes que integraron las cohortes A y B, se distribuyó de la siguiente manera: comunidad, 94 y 90 (87,9 y 80,4\%); cuidados especiales, 5 y 11 (4,7 y 9,8\%); cuidados crónicos, 3 y 5 (2,8 y 4,5\%); otros servicios del hospital, 3 y 3 (2,8\% cada uno); otro hospital, 2 y 3 (1,9 y 2,7\%), respectivamente. No hubo diferencias significativas entre las diferentes categorías ( $\mathrm{p}=0,57)$.

Como se observa en Tabla 2, en la cohorte A hubo 110 tratamientos antimicrobianos mientras en la B, hubo 117 $(p=0,36)$. En cuatro casos, los antimicrobianos se usaron en forma profiláctica y en los restantes $(n=223)$ para tratar diferentes infecciones. Dentro de éstas, la neumonía fue la indicación más frecuente: 57 en la cohorte A y 59 en la B.

El lugar de adquisición de 223 infecciones de las cohortes A y B fue: en la comunidad 78,2 y 82,1\% ( $\mathrm{p}=0,46)$, asociada a cuidados crónicos en 1,8 y 5,3\% ( $\mathrm{p}=0,18)$, de adquisición nosocomial en 20 y 12,8\% ( $\mathrm{p}=0,14)$, respectivamente.

El número de antimicrobianos utilizados osciló entre un mínimo de uno y un máximo de seis por paciente. Cada paciente en la cohorte A recibió en promedio 1,5 \pm 0,7 antimicrobianos mientras que en la B recibió $1,7 \pm$ 0,9 (diferencia no significativa).

En la Tabla 3 se puede observar el tiempo total de uso de antimicrobianos, de uso de asociación de antimicrobianos y de utilización por vía intravenosa, todos los cuales fueron significativamente menores en la cohorte A en relación a la B. Lo contrario sucedió con el tiempo de uso de vía oral, el cual fue significativamente mayor en la cohorte A.

En la Tabla 4 se comparan el modo de uso de los antimicrobianos en las cohortes de acuerdo a los criterios de la lista de verificación utilizada. En la cohorte A se observó una reducción en el uso de combinaciones de antimicrobianos, mayor proporción de paso a vía oral y de ajuste según antibiograma.

Se realizaron 98 estudios microbiológicos (43,2\% del total de infecciones). Fueron positivos 63 (64\%), 30 de 48 en la cohorte A $(62,5 \%)$ y 33 de 50 en la cohorte B (66\%). El mayor número de muestras positivas correspondió a urocultivos, seguido por cultivos de expectoración y de piel y/o tejidos blandos. No hubo diferencias entre los grupos respecto a la prevalencia de microorganismos multi-resistentes, cuatro en la cohorte A (dos muestras con SARM y dos bacilos gramnegativos (BGN) multi-resistentes y cinco en la B (3 SARM y 2 BGN multi-resistentes).

El consumo total de antimicrobianos fue de 117,7 DDD/100 días-cama. En la cohorte A el consumo fue de 46,1 DDD/100 días-cama y en la cohorte B de 71,6
Tabla 1. Características de la población según cohorte a la que pertenece

\begin{tabular}{|c|c|c|c|c|}
\hline \multirow{2}{*}{\multicolumn{2}{|c|}{ Característica }} & \multicolumn{2}{|c|}{ Cohortes, n (\%) } & \multirow[t]{2}{*}{ Valor $\mathbf{p}^{(1)}$} \\
\hline & & $A(n: 107)$ & $B(n: 112)$ & \\
\hline \multirow{2}{*}{ Sexo } & Masculino & $63(58,9)$ & $58(51,8)$ & \multirow{2}{*}{0,29} \\
\hline & Femenino & $44(41,1)$ & $54(48,2)$ & \\
\hline \multicolumn{2}{|c|}{ Tabaquismo } & $47(43,9)$ & $50(44,6)$ & 0,91 \\
\hline \multicolumn{2}{|c|}{ Enfermedad respiratoria crónica } & $31(29,0)$ & $28(25,0)$ & 0,51 \\
\hline \multicolumn{2}{|c|}{ Alcoholismo } & $16(15,0)$ & $17(15,2)$ & 0,96 \\
\hline \multicolumn{2}{|c|}{ Drogas ilegales } & $5(4,7)$ & $9(8,0)$ & 0,31 \\
\hline \multicolumn{2}{|c|}{ Diabetes mellitus } & $19(17,8)$ & $20(17,9)$ & 0,98 \\
\hline \multicolumn{2}{|c|}{ Insuficiencia cardíaca congestiva } & $17(15,9)$ & $10(8,9)$ & 0,12 \\
\hline \multicolumn{2}{|c|}{ Insuficiencia renal crónica } & $12(11,2)$ & $15(13,4)$ & 0,62 \\
\hline \multicolumn{2}{|c|}{ Diálisis crónica } & $4(3,7)$ & $4(3,6)$ & 0,95 \\
\hline \multicolumn{2}{|c|}{ Neoplasia } & $22(20,6)$ & $19(17,0)$ & 0,49 \\
\hline \multicolumn{2}{|c|}{ Inmunosupresión* } & $6(5,6)$ & $11(9,8)$ & 0,24 \\
\hline \multicolumn{2}{|c|}{ Infección por VIH } & $6(5,6)$ & $4(3,6)$ & 0,47 \\
\hline \multicolumn{2}{|c|}{ Enfermedad cerebrovascular } & $5 \quad(4,7)$ & $5(4,5)$ & 0,94 \\
\hline \multicolumn{2}{|c|}{ Otros $^{\dagger}$} & $16(15,0)$ & $23(20,5)$ & 0,16 \\
\hline
\end{tabular}

Tabla 2. Situaciones en que se prescribieron los antimicrobianos según cohortes

\begin{tabular}{lccc} 
Causa de indicación de antimicrobianos & \multicolumn{2}{c}{$\begin{array}{c}\text { Infecciones por } \\
\text { cohorte, } \mathbf{n}(\mathbf{\%})\end{array}$} & Valor $\mathbf{p}^{(1)}$ \\
& $\begin{array}{c}\text { A } \\
\text { (n }=\mathbf{1 1 0})\end{array}$ & $\begin{array}{c}\text { B } \\
(\mathbf{n}=\mathbf{1 1 7})\end{array}$ & \\
\hline Neumonías & $57(51,8)$ & $59(50,4)$ & 0,83 \\
\hline Otras respiratorias* & $9(8,2)$ & $8(6,8)$ & 0,89 \\
\hline Piel y tejidos blandos & $11(10)$ & $16(13,7)$ & 0,39 \\
\hline Infección del tracto urinario & $21(19,1)$ & $18(15,4)$ & 0,46 \\
\hline Infección del tracto urinario asociada a catéter urinario & $5(4,5)$ & $7(6,0)$ & 0,63 \\
\hline Profilaxis & $1(0,9)$ & $3(2,6)$ & 0,34 \\
\hline Otras & $6(5,5)$ & $6(5,1)$ & 0,91 \\
\hline Aclaraciones. (1) $\chi^{2}$. *Exacerbación de bronquitis crónica, sinusitis, otitis. & &
\end{tabular}

Tabla 3. Tiempo de utilización de antimicrobianos en cada cohorte

\begin{tabular}{|lccc|} 
Tiempo (días \pm DS) & \multicolumn{2}{c}{ Cohortes } & Valor de $\mathbf{p}$ \\
& A $(\mathbf{n}=\mathbf{1 1 0})$ & $\mathbf{B}(\mathbf{n}=\mathbf{1 1 7})$ & \\
Tiempo total de uso de antimicrobianos & $9,1 \pm 4,5$ & $11 \pm 6,1$ & $0,008^{(1)}$ \\
\hline Tiempo de uso de antimicrobianos asociados & $1,2 \pm 2,5$ & $3,2 \pm 5,9$ & $0,01^{(2)}$ \\
\hline Tiempo de uso de antimicrobianos intravenosos & $4,2 \pm 2,8$ & $8,6 \pm 6,9$ & $<0,0001^{(2)}$ \\
\hline Tiempo de uso de antimicrobianos orales & $4,8 \pm 3,9$ & $3,3 \pm 4,0$ & $0,004^{(1)}$ \\
\hline Aclaraciones. DS: desvío estándar. (1)test de T. (2) prueba de Mann-Whitney. &
\end{tabular}


Tabla 4. Utilización de los criterios de uso racional de antimicrobianos según cohortes

\begin{tabular}{lccc}
$\begin{array}{l}\text { Criterios de uso racional } \\
\text { de antimicrobianos }\end{array}$ & A $(\mathbf{n}=\mathbf{1 1 0})$ & $\mathbf{B}(\mathbf{n}=\mathbf{1 1 7})$ & Valor $\mathbf{p}^{(1)}$ \\
\hline Pasaje a la vía oral & $80(73,4)$ & $38(33,3)$ & $<0,0001$ \\
\hline Adaptación al antibiograma & $31(64,6)$ & $12(24)$ & 0,001 \\
\hline Asociación de antimicrobianos & $29(26,6)$ & $47(41,2)$ & 0,03 \\
\hline Aclaraciones. ${ }^{(1)} \chi^{2}$. & & & \\
\hline
\end{tabular}

Tabla 5. Comparación de consumo de antimicrobianos por vía parenteral en cohorte la B respecto a la cohorte $\mathrm{A}$

\begin{tabular}{|lcccc|}
\hline Antimicrobiano & $\begin{array}{c}\text { Cohorte B } \\
\text { DDD/100 } \\
\text { días-cama }\end{array}$ & $\begin{array}{c}\text { Cohorte A } \\
\text { DDD/100 } \\
\text { días-cama }\end{array}$ & $\begin{array}{c}\text { Diferencia } \\
\text { DDD/100 } \\
\text { días-cama }\end{array}$ & Diferencia \% \\
\hline Penicilina & 17,5 & 12,4 & $-5,1$ & -29 \\
\hline Ampicilina & 2,4 & 0,8 & $-1,6$ & -67 \\
\hline Ampicilina/sulbactam & 14,6 & 10 & $-4,6$ & -31 \\
\hline Cefradina & 0,5 & 0,2 & $-0,3$ & -60 \\
\hline Ceftriaxona & 4,5 & 1,2 & $-3,3$ & -73 \\
\hline Ceftazidima & 1,1 & 0,4 & $-0,7$ & -64 \\
\hline Ciprofloxacina & 2,3 & 0,5 & $-1,8$ & -78 \\
\hline Clindamicina & 2,6 & 1,4 & $-1,2$ & -46 \\
\hline Metronidazol & 0,4 & 0,05 & $-0,35$ & -87 \\
\hline Amikacina & 0,7 & 0,3 & $-0,4$ & -57 \\
\hline Vancomicina & 0,6 & 0,05 & $-0,55$ & -92 \\
\hline Carbapenems & 0,6 & 0,2 & $-0,4$ & -67 \\
\hline Total & 47,8 & 27,5 & $-20,3$ & -43 \\
\hline
\end{tabular}

Tabla 6. Comparación de consumo de antimicrobianos por vía oral en la cohorte B respecto a la $\mathbf{A}$

\begin{tabular}{|lcccr|} 
Antimicrobiano & $\begin{array}{c}\text { Cohorte B } \\
\text { DDD/100 } \\
\text { días-cama }\end{array}$ & $\begin{array}{c}\text { Cohorte A } \\
\text { DDD/100 } \\
\text { días-cama }\end{array}$ & $\begin{array}{c}\text { Diferencia } \\
\text { DDD/100 } \\
\text { días-cama }\end{array}$ & $\begin{array}{c}\text { Diferencia } \\
\%\end{array}$ \\
Amoxicilina & 0,5 & 2,8 & $+2,3$ & +460 \\
\hline Amoxicilina/inhibidor de $\beta$-lactamasa & 14,6 & 10,2 & $-4,4$ & -30 \\
Cefradina & 0,05 & 0,4 & $+0,35$ & +700 \\
Cefuroxima & 1,1 & 1,2 & $+0,1$ & +9 \\
Ciprofloxacina & 0,5 & 0,6 & $+0,1$ & +20 \\
Claritromicina & 5,9 & 1,3 & $-4,6$ & -78 \\
Clindamicina & 0,6 & 1,4 & $+0,8$ & +133 \\
Cotrimoxazol & 0,5 & 0,7 & $+0,2$ & +40 \\
Total & 23,8 & 18,6 & $-5,2$ & -22 \\
\hline
\end{tabular}

DDD/100 días-cama. La reducción en el consumo de antimicrobianos en la cohorte A respecto de la B, fue de 35,6\%. En las tablas 5 y 6 se muestran y comparan el consumo de todos los antimicrobianos por vía parenteral y oral.

Hubo una reducción en el consumo de todos los antimicrobianos por vía parenteral en la cohorte A. El consumo de cefalosporinas de tercera generación fue menor en la cohorte A respecto a la B (reducción en 73\% de ceftriaxona, y 64\% de ceftazidima). También se verificó una reducción en el consumo total de quinolonas de 61\%; sin embargo, esta reducción se produjo en el consumo parenteral (78\%), mientras que el consumo por vía oral fue mayor en la cohorte A (20\%). Se observan resultados similares en el consumo de clindamicina, reducción de $12,5 \%$ en el consumo total, con menor consumo por vía parenteral y mayor consumo oral en la cohorte A. Si bien el consumo de antimicrobianos dirigidos a microorganismos multi-resistentes fue bajo en ambos grupos, fue menor aún en la cohorte A respecto a la $\mathrm{B}$, vancomicina (reducción de 92\%) y carbapenem (reducción de 67\%).

Los aminoglucósidos (AG) fueron utilizados en un total de 11 infecciones, cinco de la cohorte A y seis de la B. Sólo se empleó amikacina. Una pauta de monodosis se utilizó en cuatro pacientes de la cohorte A(dos infecciones del tracto urinario y dos neumonías intrahospitalarias) $\mathrm{y}$ en ninguno de las seis infecciones de la cohorte B (dos infecciones del tracto urinario, tres neumonías, una infección de tejidos blandos).

Respecto al consumo de antimicrobianos por vía oral, en la cohorte A fue mayor en casi todos, excepto en amoxicilina asociada a un inhibidor de ß-lactamasa y claritromicina. Amoxicilina/inhibidor de ß-lactamasa (sulbactam o ácido clavulánico) fue utilizada predominantemente en el cambio a vía oral en el curso del tratamiento de una neumonía. Se asoció claritromicina a un ß-lactámico en el tratamiento de 10 de 57 (17,5\%) neumonías de la cohorte A y en 20 de 59 (34\%) neumonías de la cohorte B. El tiempo de uso de claritromicina fue significativamente menor en la cohorte A respecto de la $\mathrm{B}$; 3,0 $\pm 1,6$ días y 9,0 \pm 4,2 días, respectivamente ( $\mathrm{p}=$ 0,001, test de T).

Se calcularon los costos relacionados al uso de antimicrobianos considerando el costo del fármaco y los insumos relacionados a la administración parenteral. En la cohorte A hubo una reducción de 55\% del costo total respecto a la B y una reducción de $30 \%$ en unidades dispensadas. La reducción de costos fue fundamentalmente a expensas de los antimicrobianos parenterales; 57,7\% menor en la cohorte A.

La estadía media hospitalaria y su desviación estándar fue de $16 \pm 13$ días para la cohorte A, vs $18 \pm 16$ días para la cohorte $\mathrm{B}(\mathrm{p}=0,3$, test de $\mathrm{T})$. Hubo 7 muertes en la cohorte A y 9 en la cohorte B. 


\section{Discusión}

La aplicación de una estrategia sencilla basada en una lista de verificación conteniendo un recordatorio de medidas para racionalizar el uso de antimicrobianos y supervisión de especialistas capacitados, impactó favorablemente en el uso de antimicrobianos en nuestro establecimiento, sin un impacto negativo en la mortalidad ni en la estadía media hospitalaria. Esta estrategia tuvo un fuerte componente educativo, de acuerdo a su ámbito de aplicación en un hospital universitario, lo que favorece la adhesión a las recomendaciones.

A pesar que el diseño del estudio no fue pareado, la población analizada en las cohortes fue homogénea en sus características basales. Tampoco hubo diferencias significativas en el tipo de infecciones y el lugar de adquisición de las mismas.

Sí bien el número de antimicrobianos empleados por cada infección fue similar en ambas cohortes, el tiempo total de uso y el tiempo de uso por vía intravenosa fue significativamente menor en la cohorte A. El uso de antimicrobianos por tiempos excesivamente prolongados es una causa frecuente de uso inapropiado de estos fármacos. Esta conducta ha sido descrita frecuentemente en infecciones respiratorias, a pesar que existe un cuerpo creciente de evidencias respecto a la eficacia de tratamientos acortados en estas infecciones ${ }^{26,27}$.

El uso de terapia antimicrobiana asociada fue significativamente menor en la cohorte A respecto a la B (26,6 vs 41,2\%). El uso de terapia antimicrobiana combinada no se justifica en la mayoría de las infecciones. Esta estrategia tiene un rol bien definido en contextos específicos. Es el caso del inicio de terapia empírica en pacientes con infecciones graves, con el objetivo de lograr sinergismo, ampliar el espectro antimicrobiano así como evitar la emergencia de resistencia intratratamiento.

El paso a vía oral se realizó en una proporción de pacientes significativamente mayor de la cohorte A respecto a la B (73,4 vs 33,3\%). Estos resultados son similares a resultados de estudios de "switch" terapia reportados en la literatura científica internacional ${ }^{28-32}$. Existe una tendencia generalizada a completar los cursos de antimicrobianos en pacientes hospitalizados por vía intravenosa, sin considerar el paso a vía oral o realizándolo tardíamente. El concepto de terapia secuencial antimicrobiana (TSA) significa el paso precoz de la vía intravenosa a la vía oral en pacientes estabilizados con mejoría clínica de su cuadro infeccioso, sin trastornos gastrointestinales que limiten su tolerancia y/o absorción y con una alternativa terapéutica oral adecuada a la infección a tratar. El paso a vía oral se realiza al mismo antimicrobiano (si se dispone) o a uno de espectro simi$\operatorname{lar}^{33}$. Además, cuando se ha aislado el microorganismo, es la oportunidad de ajustar el antimicrobiano al perfil de susceptibilidad (término en inglés: “de-escalation”). El beneficio de la TSA radica en disminuir los problemas asociados a la presencia de accesos venosos y tiene la conveniencia de reducir los costos derivados de una internación prolongada.

Se destaca la baja proporción de estudios para confirmación microbiológica realizados $(43,2 \%)$, de los cuales $64 \%$ resultaron en la identificación del agente etiológico. La adaptación a la susceptibilidad antimicrobiana con reducción del espectro se realizó más frecuentemente en la cohorte A respecto a la B (64,6 vs 24\%). Esta estrategia es uno de los pilares de uso apropiado de antimicrobianos dado que implica reducir el uso de antimicrobianos de amplio espectro así como, en general, es posible evitar el uso de terapia antimicrobiana combinada, disminuyendo el riesgo de selección de resistencia y los costos adicionales $^{34}$.

El consumo total de antimicrobianos en el período fue de 117,7 DDD/100 días-cama, por lo que el consumo de este grupo de fármacos en los servicios de medicina interna de nuestro hospital es comparable al informado para otros hospitales con características similares ${ }^{35}$. En cuanto al consumo de antimicrobianos por grupo, los resultados muestran una reducción en la cohorte A respecto a la B, tanto en el consumo total como en el consumo discriminado por vía de administración.

El consumo total de quinolonas y clindamicina fue menor en la cohorte A; sin embargo, esta reducción se hizo a expensas de la administración parenteral, mientras que el consumo por vía oral fue mayor en esta cohorte. Este hecho probablemente refleja la promoción del uso de estos antimicrobianos por vía oral dada su excelente biodisponibilidad.

Existe una relación bien establecida entre el uso de determinados antimicrobianos (cefalosporinas de tercera generación y quinolonas) con la mayor incidencia de resistencia de algunos microorganismos (BGN productores de ß-lactamasas de espectro extendido, BGN no fermentadores multi-resistentes), así como las políticas que implican restricción de estos antimicrobianos tienen un impacto positivo tanto sobre la colonización como la infección por estos microorganismos ${ }^{4,5,10,19,21,22}$. También se ha reportado una menor incidencia de diarreas por Clostridium difficile como resultado de la implementación de estrategias de uso apropiado de antimicrobianos, con restricción del uso de cefalosporinas y clindamicina ${ }^{14,16}$. La aplicación de una lista de verificación, incluyendo el criterio de considerar el cambio a un antimicrobiano alternativo a la prescripción de cefalosporinas de tercera generación, quinolonas, vancomicina y carbapenem, permitió obtener una reducción en el consumo de estos antimicrobianos.

Dada las características farmacocinéticas/farmacodi- 
námicas de los aminoglucósidos se recomienda su uso en monodosis diaria en la mayoría de las infecciones donde están indicados. En este estudio, si bien la utilización de aminoglucósidos fue baja, fueron utilizados en pauta de monodosis en 4 de 5 infecciones de la cohorte A y en ninguna de 6 infecciones de la cohorte $B$.

La principal prescripción de claritromicina se observó asociada a ß-lactámicos en pacientes con neumonías. En la cohorte A hubo un menor consumo de dicho antimicrobiano como consecuencia de su menor prescripción y reducción en el tiempo de uso. En esta cohorte se desestimó el uso sistemático de la asociación de ß-lactámicos y macrólidos en todas las neumonías, restringiéndose su uso a aquellas de mayor gravedad.

Hubo un ahorro en costos económicos mayor a 50\% en la cohorte A, debida fundamentalmente a la reducción de los antimicrobianos de uso parenteral. Esto pudo tener un impacto, más allá de lo económico, en la reducción de uso de vías venosas y las complicaciones relacionadas, variables no medidas en nuestro estudio.

La estadía media hospitalaria y la mortalidad fueron similares en ambas cohortes. En estos resultados, probablemente influyeron otras variables, además de la infección, como las co-morbilidades descritas en la población.

La racionalización en el uso de antimicrobianos implica tener en cuenta parámetros farmacocinéticos y farmacodinámicos, así como realizar monitoreo de concentraciones en sangre de algunos antimicrobianos, para adecuar las pautas posológicas, aspectos que no fueron evaluados en nuestro estudio (excepto en el uso de aminoglucósidos en monodosis).

Por otra parte, el objetivo final de la implementación de políticas de uso racional de antimicrobianos es disminuir la selección de resistencia bacteriana y se recomienda que este tipo de estudios evalúen la eficacia de las medidas e intervenciones a través del impacto en la incidencia de microorganismos resistentes, tanto en colonización como infección. Este resultado no puede evaluarse a través del presente estudio lo que constituye una de sus limitaciones. El diseño del mismo, basado en la aplicación de medidas en un área restringida del hospital y por un tiempo limitado, excluye la posibilidad de medir este impacto.

\section{Conclusiones}

La implementación de un programa de uso racional de antimicrobianos basado en la aplicación de una lista de verificación que incluía criterios validados, sumado a la interacción de expertos con los médicos a cargo de la prescripción (componente educativo), permitió reducir el consumo de antimicrobianos, optimizando su uso y disminuir los costos, sin un impacto negativo en la estadía hospitalaria y mortalidad. Es recomendable la integración de un equipo interdisciplinario para el desarrollo de un programa de uso apropiado de antimicrobianos en nuestro hospital.

Agradecimientos: a Q.F. Carmen Duré, Q.F. Anna Boronat. Dpto. de Farmacia del Hospital de Clínicas.

\section{Resumen}

Introducción: Los programas de uso racional de antimicrobianos tienen la finalidad de optimizar la terapia antimicrobiana y minimizar la aparición de resistencia bacteriana. Con el objetivo de optimizar el uso de antimicrobianos se implementó un programa educativo basado en la aplicación de una lista de verificación (check list) conteniendo criterios establecidos de uso racional de estos fármacos. Método: Se realizó un estudio de cohortes no pareadas en el Departamento de Medicina Interna, durante tres meses. Se comparó una cohorte prospectiva (A) en que se aplicó la lista de verificación, con una cohorte retrospectiva (B) con prescripción de acuerdo a la práctica clínica habitual. Resultados: Se incluyeron 227 prescripciones de antimicrobianos. En la cohorte A, hubo mayor proporción de paso a vía oral y adecuación del antimicrobiano al antibiograma y menor uso de asociación de antimicrobianos, con respecto a la cohorte $\mathrm{B}$. El consumo total de antimicrobianos fue de 117,7 DDD/100 días-cama (Dosis Diaria Definida). El consumo en las cohortes A y B fue de 46,1 DDD/100 días-cama y 71,6 DDD/100 días-cama respectivamente (reducción de un 35,6\%). También hubo una reducción en el consumo de ceftriaxona, ceftazidima, quinolonas, vancomicina y carbapenem. Los costos se redujeron en $55 \%$. No hubo diferencias en la estadía media hospitalaria. Conclusiones: La aplicación de una estrategia educativa basada en una lista de verificación permitió optimizar el uso de antimicrobianos.

\section{Referencias}

1.- John J F Jr., Fishman N O. Programmatic role of the infectious diseases physician in controlling antimicrobial costs in the hospital. Clin Infect Dis 1997; 24: 471-85.

2.- Willemsen I, Groenhuijzen A, Bogaers D, Stuurman A, van Keulen P, Kluytmans J. Appropriateness of antimicrobial therapy measured by repeated prevalence surveys. Antimicrob Agents Chemother 2007; 51: 864-7.

3.- Monroe S, Polk R. Antimicrobial use and bacterial resistance. Curr Opin Microbiol 2000; 3: 496-501.

4.- Muller A A, Mauny F, Bertin M, Cornette C, López-Lozano J M, Viel J F, et al. Relationship between spread of methicillin-resistant Staphylococcus aureus and antimicrobial use in a French university hospital. Clin Infect Dis 2003; 36: 971-8.

5. Polk R E, Johnson C K, McClish D, Wenzel R P, Edmond M B. Predicting hospital rates of fluorquinolone-resistant Pseudomonas aeruginosa from fluorquinolone use in US-hospitals and their surrounding communities. Clin Infect Dis 2004; 39: 497-503. 
6.- Cosgrove S E, Sakoulas F, Perencevich E N, Schwaber M J, Karchmer A W, Carmeli Y. Comparison of mortality associated with methicillin-resistant and methicillin-susceptible Staphylococcus aureus bacteriemia: a metaanalysis. Clin Infect Dis 2003; 36: 53-9.

7.- Díaz-Granados C A, Zimmer S M, Klein M, Jeringan J A. Comparison of mortality associated with vancomycin-resistant and vancomycin-susceptible enterococcal bloodstream infections: a meta-analysis. Clin Infect Dis 2005; 41: 327-33.

8.- Engemann J J, Carmeli Y, Cosgrove S E, Fowler V G, Bronstein M Z, Trivette S L, et al. Adverse clinical and economic outcomes attributable to methicillin resistance among patients with Staphylococcus aureus surgical site infection. Clin Infect Dis 2003; 36: 592-8.

9.- Cosgrove $\mathrm{S}$ E. The relationship between antimicrobial resistance and patient outcomes: mortality, lenght of hospital stay, and health care costs. Clin Infect Dis 2006; 42 (Suppl 2): S82-9.

10.- Cosgrove S E, Kaye K S, Eliopoulous G M, Carmeli Y. Health and economic outcomes of the emergence of third-generation cephalosporin resistance in Enterobacter species. Arch Intern Med 2002; 162: 185-90.

11.- Palacio R, Alonso R, Romero S, Bazet C. Costos de infecciones intrahospitalarias por Staphylococcus aureus; meticilino resistente vs meticilino sensible. Rev Panam Infectol 2006; 8: 33-8.

12.- Lesprit P, Brun-Buisson C. Hospital antibiotic stewardship. Curr Opin Infect Dis 2008; 21: 344-9.

13.- Dellit T H, Owens R C, MaGowan J E, Gerding D N, Weinstein R A, Burke J P, et al. Infectious Diseases Society of America and the Society for Healthcare Epidemiology of America Guidelines for developing and Institucional Program to Enhance Antimicrobial Stewardship. Clin Infect Dis 2007; 44: 159-77.

14. López-Medrano F, San Juan R, Serrano O, Chaves F, Lumbreras C, Lizasoaín M, et al. Impact of a non-compulsory antibiotic control program (PACTA): cost reductions and decreases in some nosocomial infections. Enferm Infecc Microbiol Clin 2005; 23: 186-90.

15.- Zillich A J, Sutherland J M, Wilson S J, Diekema E J, Ernst E J, Vaughn T E, et al. Antimicrobial use control measures to prevent and control antimicrobial resistance in US hospitals. Infect Control Hosp Epidemiol 2006; 27: 1088-95.
16.- Carling P, Fung T, Killion A, Terrin N, Barza M. Favorable impact of a multidisciplinary antibiotic management program conducted during 7 years. Infect Control Hosp Epidemiol 2003; 24: 699-706

17.- Solomon DH, Van Houten L, Glynn RJ, Baden L, Curtis K, Schrager H, et al. Academia detailing to improve use of broad-spectrum antibiotics at an academic medical center. Arch Intern Med 2001; 161: 1897-902.

18.- Ruttimann S, Keck B, Hartmeier C, Maetzel A, Bucher H C. Long-term antibiotic cost savings from a comprehensive intervention program in a Medical Department of a University-affiliated teaching hospital. Clin Infect Dis 2004; 38: 348-56.

19.- Aspinall S L, Metlay J P, Maselli J H, Gonzales R. Impact of hospital formularies on fluorquinolone prescribing in emergency departments. Am J Manag Care 2007; 13: 241-8.

20.- Ranji S R, Steinman M A, Shojania K G, Gonzales R. Interventions to reduce unnecessary antibiotic prescribing. A systematic review and quantitative analysis. Med Care 2008; 46: 847-62.

21.- Bantar C, Sartori B, Vesco E, Heft C, Saúl M, Salamone F, et al. A hospital wide intervention program to optimize the quality of antibiotic use: Impact on prescribing practice, antibiotic consumption, cost savings, and bacterial resistance. Clin Infect Dis 2003; 37: 180-6.

22.- Lipworth A D, Hyle E P, Fishman N O, Nachamkin I, Bilker W B, Marr A M, et al. Limiting the emergence of extended-spectrum ß-lactamase-producing Enterobacteriaceae: Influence of patient population characteristics on the response to antimicrobial formulary interventions. Infect Control Hosp Epidemiol 2006; 27: 279-86.

23.- Giachetto G, Alvarez C, Arnaud H, Bruno P, Da Silva E, De Salterain H, et al. Uso de antibióticos en servicios de internación pediátrica. Rev Med Uruguay 2001; 17: 55-61.

24.- Giachetto G, Martínez A, Pírez MC, Algorta G, Banchero P, Camacho G, et al. Vigilancia del uso de antibióticos en el Hospital Pediátrico del Centro Hospitalario Pereira Rossell: susceptibilidad antimicrobiana; gasto y consumo de antibióticos. Rev Med Uruguay 2003; 19 : 208-15.

25.- WHO Collaborating Centre for Drug Statistics Methodology. Guidelines for ATC classification and DDD assignment 2010. Oslo, 2009. Disponible en: www.whocc.no/atcddd (accedido en febrero 2010).
26.- Chastre J, Wolff M, Fagon J-Y, Chevret S, Thomas F, Wermert D, et al. Comparision of 8 vs 15 days of antibiotic therapy for ventiladorassociated pneumonia in adults. JAMA 2003; 290: 2588-98.

27.- Moussaoui R, de Borgie C, van den Broek P, Hustinx WN, Bresser P, vsn den Berk GEL, et al. Effectiveness of discontinuing antibiotic treatment alter three days versus eight days in mild to moderate-severe community acquired pneumonia: randomised, double blind study. $\mathrm{Br}$ Med J 2006; 332: 1355-61.

28.- Sevinc F, Prins J M, Koopmans R P, Langendijk P N J, Bossuyt P M M, Dankert J, et al. Early switch from intravenous to oral antibiotics: guidelines and implementation in a large teaching hospital. J Antimicrob Chemother 1999; 43: 601-6.

29.- Rhew D C, Tu G S, Ofman J, Henning J M, Richards M S, Weingarten S R. Early switch and early discharge strategies in patients with community-acquired pneumonia: a meta-analysis. Arch Intern Med 2001; 161: 722-7.

30.- McLaughlin C M, Bodasing N, Boyter A C, Fenelon C, Fox J G, Seaton R A. Pharmacyimplemented guidelines on switching from intravenous to oral antibiotics: an intervention study. QJM 2005; 98: 745-52.

31.- Mertz D, Koller M, Haller P, Lampert M L, Plagge H, Hug B, et al. Outcomes of early switching from intravenous to oral antibiotics on medical wards. J Antimicrob Chemother 2009; 64: 188-99.

32.- Waagsbo B, Sundoy A, Paulsen E Q. Reduction of unnecessary IV antibiotic days using general criteria for antibiotic switch. Scand J Infect Dis 2008; 40: 468-73.

33.- Aguado J M, Fortún J. Guía de recomendaciones en la Terapia Secuencial Antibiótica (TSA). En: Guías Clínicas de la Sociedad Española de Enfermedades Infecciosas y Microbiología clínica (SEIMC) 2006. Disponible en: www.seimc.org Accedido:

34.- Glowacki R C, Schwartz D N, Itokazu G S, Wisniewski P, Weinstein R A. Antibiotic combinations with redundant antimicrobial spectra: clinical epidemiology and pilot intervention of computer-assisted surveillance. Clin Infect Dis 2003; 37: 59-64.

35.- Kuster S P, Ruef C, Ledergerber B, Hintermann A, Deplazes C, Neuber L, et al. Quantitative antibiotic use in hospitals: comparision of measurements, literatura review, and recommendations for standard of reporting. Infection 2008; 36: 549-59. 